\title{
Trends of Tuberculosis in Somaliland's Young Children after the Conflict and the Role Khat Marfishes Play Its Transmission
}

Mohamed Hassan*

Department of Statistics, United Arab Emirates University, Al-Ain, UAE

\begin{abstract}
After the fall of the central government and the emergence of the recurrent civil conflict in Somalia, many new sociocultural phenomenons that have appeared across the country contributed to the spread of many infectious diseases including TB. One of these social phenomena is a wide spread use of the illicit drug Khat which is predominately used by the Somali males. Mostly khat is chewed in small overcrowded, unhygienic and unventilated makeshift huts known as Marfishes. TheseMarfishes becamethelaunch padandthebreeding grounds of many infectious diseases that have affected the lives of many Somalis including children. Under-five mortality in somalia is estimated at 200 deaths per 1000 births, which is one of the highest in the world. Approximately one third of these are neonatal deaths, occurring during the first month of life, pneumonia and diarrhea are the main killers each contributing 20-25 percent of all under-five mortality. While thesediseasesstill remained the top major killers, communicable diseases including TBarealsoaleading cause of death. This paper investigates the incidence and the trends of tuberculosis among the Somali children by using time series statistical models.
\end{abstract}

Keywords: Children under five years old; Tuberculous; Time series; Holt-winters exponential smoothing; Khat Marfishes

\section{Introduction}

Tuberculosis remains a serious public health problem worldwide, and in particular, in the developing countries [1]. It is one of the leading causes of mortality and morbidity across all age groups throughout the world, especially in the developing countries [2]. Over 95\% of TB deaths occur in low-middle income countries, and it is among the top 5 causes of death for women aged 15 to 44 [1], more references about how TB is spreading over the globe, see, for example [3-5]. As $\mathrm{TB}$ is an airborne infectious disease, transmission is associated with the contact of an infectious TB case and the intimacy of that contact $[6,7]$. In 2014 alone, more than 9.6 million people fell ill with TB including an estimated one million children. During that year, 1.5 million died for the disease of which 140000 or almost $15 \%$ were children [1], and in 2006 that number was estimated 11\% [8]. In areas with a high incidence of TB, children contribute $15-40 \%$ of the total case load and experience considerable mortality $[7,9,10]$. Among children, the greatest numbers of TB cases are seen in those less than 5 years of age, and in adolescents older than 10 years of age [11]. Childhood tuberculosis (TB) reflects recent transmission, and it is an important indicator of public health success in interrupting and preventing TB transmission. High rates of childhood TB indicates that opportunities for preventing TB are being missed [12]. In endemic countries, the focus is mainly on adults with smear positive TB while TB in children receives little attention $[13,14]$. Despite the fact that one million child TB cases were reported in 2014, TB disease and latent TB infection (LTBI) frequencies in children are dramatically underestimated. The sum total of MDR-TB child cases that have ever been reported in the literature is just $2 \%$ of those that they estimate occurred globally in the year 2010 alone [15]. In their study [15], they have mentioned multiple factors that would explain the present sizeable underestimate of the incidence of TB disease in children. Firstly, Young children experience the highest risks of severe disease and death once infected, but are the least likely to be confirmed bacteriologically as TB cases. This reflects the fact that children are more likely than adults to have paucibacillary disease and those young children (less than 5 years old) cannot expectorate sputum, preventing microbiological diagnosis. Secondly, all currently available microbiological tests have very low sensitivity for child TB disease. Applying these calculated risks, their estimates showed that around $1,000,000$ children developed TB disease in 2010 , among which 32,000 had MDR-TB. TB disease among children is much more prevalent in developing countries, where resources for TB control are scarce, than in industrialized countries [16]. In resource-limited settings, many deaths in children with pneumonia are TB-related [9,17], and it is more common in countries with failed states like Somalia where TB is more prevalent. In the first quarter of 2013 alone, 3300 new TB cases were detected in that country, at a time new drug resistant tuberculosis is emerging in many parts of the world. In a nationwide survey in 2011 [18] multidrug-resistant tuberculosis (MDR TB) was found in $5.2 \%$ and $40.8 \%$ of patients with new and previously treated $\mathrm{TB}$ respectively. These levels of drug resistance are among the highest ever documented in Africa and the Middle East. The estimated per capita incidence rate for TB in Somalia for 2013 was 253 per 100,000 (WHO). Under-five mortality in somalia is estimated at 200 deaths per 1000 births, which is one of the highest in the world. Approximately one third of these are neonatal deaths, occurring during the first month of life, pneumonia and diarrhea are the main killers each contributing 20-25 percent of all under-five mortality. While these diseases still remained the top major killers, communicable diseases including TB are also a leading cause of death.

*Corresponding author: Mohamed Hassan, Department of Statistics, United Arab Emirates University, Al-Ain, PO Box 15551, UAE, Tel: +97137673333; E-mail: myusuf@uaeu.ac.ae

Received February 09, 2018; Accepted February 23, 2018; Published February 26, 2018

Citation: Hassan M (2018) Trends of Tuberculosis in Somaliland's Young Children after the Conflict and the Role Khat Marfishes Play Its Transmission. J Biom Biostat 9: 397. doi: 10.4172/2155-6180.1000397

Copyright: ( $) 2018$ Hassan M. This is an open-access article distributed under the terms of the Creative Commons Attribution License, which permits unrestricted use, distribution, and reproduction in any medium, provided the original author and source are credited. 


\section{The Role of the Social Determinants and the Over- crowded Khat Marfishes on the Spread of the Diseases}

It has been known for many decades that tuberculosis is a social disease that is highly correlated with environmental risk factors and cultural backgrounds among people sharing a particular community. Many conditions that increase the risk of TB infection arise from the circumstances in which people living in a particular community are born, grow, live, work and age [19]. Social determinants of health are mostly responsible for health inequities between and within countries (WHO). Because of the social connections between an index patient and contacts, social determinants of infectious diseases in the world become a public health priority [20]. These determinants which may vary by geographical region and cultural background can be used to shed light the role social processes could play the spread of tuberculosis globally as well as nationally to develop effective prevention strategies. Lack of consideration for the entrenched cultures and their embedded social determinants may predispose general population high rates of $\mathrm{TB}$ infections. Environmental risk factors such as indoor air pollution, and overcrowded living conditions exacerbate TB risks in some countries like Somalia where crowding tends to be associated with other socioeconomic and living conditions [14]. Proximity and persistence of contact are major determinants of transmission, and those living within the same household are at higher risk than casual contacts [2]. Proximate risk factors include those that directly increase exposure to infectious droplets. Among household contacts, young children are at increased risk of getting infection from an index case [21].

In Somalia after the fall of the central government in 1991, many new sociocultural phenomenons that have appeared across the country contributed to the spread of many infectious diseases including TB. One of these social phenomena is a wide spread use of the illicit drug khat ${ }^{1}$ which is predominately used by the Somali males. Binge khat chewing in small overcrowded, unhygienic and unventilated makeshift huts known as Marfishes mostly concentrated in small villages and big cities become a common habit across the regions inhabited by ethnic Somalis. These huts are increasingly becoming breeding grounds for many infectious diseases including Mycobacterium tuberculosis. Khat Marfishes were also common where Somalis reside in the UK before it was banned in the country in 2014; currently its trade, possession and use are illegal in many countries including UK. Heathrow airport served as a hub and a distribution center to the UK and to many other European and North American countries [22]. In a report about the health and social impacts of khat use released by Brent council in the UK indicated that, among consequences attributed to khat use are infectious diseases, such as TB or pneumonia, contracted as a result of taking khat in confined and poorly ventilated spaces in mafrishes ${ }^{2}$. The Somali community in the UK believes that, the number of Tuberculosis patients that health authorities blamed on the enclosed Mafrishes which they say was conducive for the spread of the airborne disease has dramatically declined after it was banned in the UK.

\section{Overcrowded Khat Marfishes and the Spread of Tuberculosis among Somali Children}

There is accumulating compelling evidence that overcrowded khat Marfishes contributed the dissemination of tuberculosis (TB) amongst the Somalis and created perfect conditions on its transmission. There

${ }^{1}$ Khat (Catha edulis) is a large green shrub mainly cultivated in the highlands of the Horn of Africa and Southern Arabia.

${ }^{2}$ https://www.brent.gov.uk/media/2025436/0702012- khat1.pdf is significant evidence about the adverse effects of overcrowding and unhygienic, unventilated buildings on the spread of tuberculosis. An ecological analysis of childhood tuberculosis in New York [23,24] showed that the average level of notifications is correlated with overcrowding [25]. In an another study it has also been found for each $1 \%$ increase in the numbers living in overcrowded accommodation the average notification rate for tuberculosis increased by $12 \%$ [25]. Bhatti et al. concluded in their studies that, there was a strong association of tuberculosis risk with overcrowding [26]. Baker et al. conducted the first study of its type in New Zealand to look at the effects of household crowding on TB [27]. They found that, TB risk of infection is increased by household crowding. Their investigation is focused among the 40 years or younger age group in New Zealand-born population. They found that on average for every $1 \%$ increase in household crowding in a neighborhood the expected number of TB cases will increase by $8 \%$, holding other variables constant. Other studies also show overcrowding, poor ventilation and lack of screening for $\mathrm{TB}$ in the prisons made these places breeding grounds and incubators for TB. For example, in Russia’s overcrowded prisons populated largely by young and poor people, TB incidence leapt toward 5,000 cases per 100,000 during the late 1990s and early 2000 [28].

In Somalia, many adult males who are obsessed with chewing khat in these overcrowded places became a source of TB infection to their families including children. Those fathers are living with their families and mostly share the master bedroom with multiple children. Many of those children had close contact with a father who had active tuberculosis for a long period of time. Risk factors of child infection are younger age, birth in a high prevalence country and prolonged close contact with an index case. Many studies demonstrated a very strong association between parental TB infection status and infection status of preschool-age children [29]. Exposure to an adult with pulmonary TB was reported to increase mortality by $70 \%$ in children under 5 years of age in high-burden settings and by eightfold when the mother had TB [9]. Risk factors for pediatric infection included exposure to a parent who had active tuberculosis and that risk of infection increases if their father had a current TB infection. Unless more attention is given to the linkages between Marfishes and communicable diseases in Somalia, the spread of infectious diseases in the country will be very difficult to control. Any appropriate preventive strategy should depend on funding campaigns that are designed to curbing binge khat chewing in these places, and a strong emphasis should be placed on facilitating the socio-cultural factors that inhibit this habit. While the currently focus of TB control is based on prompt case detection and effective treatment, incubators for tuberculosis like the levels of crowding also need to be considered. Reducing the number of breeding grounds could play an important role in controlling the spread of this disease. Thus, progress towards the elimination of tuberculosis needs to put more emphasis not only on diagnosis and treatment of the latent infection but also on environmental interventions based on controlling breeding grounds.

\section{Methods}

\section{Time series analysis of $\mathrm{Tb}$ data collected from the under five years children in Somaliland}

Proactive infection control measures are the best way to prevent the spread of tuberculosis. Those measures cannot be implemented without early detection of people in need of the disease treatment which relies on higher quality forecasts and effective strategic planning. Forecasting incidence of communicable diseases and in particular (TB) plays an important role in planning, control, developing intervention programs 
and allocating resources. Early prediction of its trends also helps to choose the most appropriate and timely interventions before the outbreak of the disease occurs and avoids any lapses in controlling the spread of the disease. Lack of relevant information about the spread of the TB could also pose significant challenges to those providing services to the prevention, treatment, and control of the disease. Thus, any TB control plan designed to ensure effective prevention must include continuous monitoring and surveillance based on broad understanding of the local living conditions and better prediction methods. So far, a number of studies were conducted to investigate the temporal structure of these diseases. Those studies used different forecasting time series methods to predict the morbidity and mortality of the disease [30-34]. Time series modeling is fundamental in predicting and controlling the spread of many infectious diseases. Most common time series models include various forms of weighted smoothing methods, decomposition techniques which decompose the series into trend, seasonal, cyclic and random components, Box-Jenkins models, time series regression and curves fitting. In this section TB trends among the under-five year old

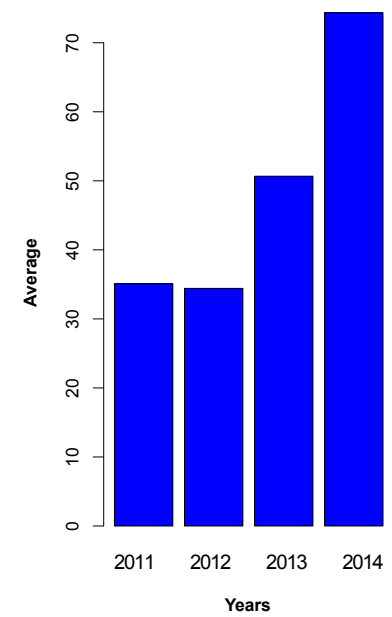

(b)

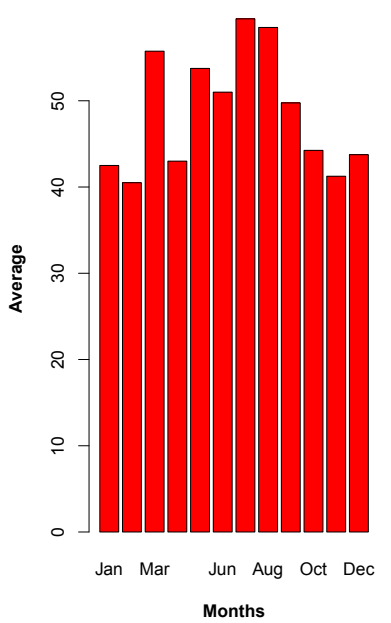

Figure 1: Under five years TB data 2011-2014 (a) Yearly averages (b) Monthly averages.

Below 5 Year TB by Region in 2011-2014

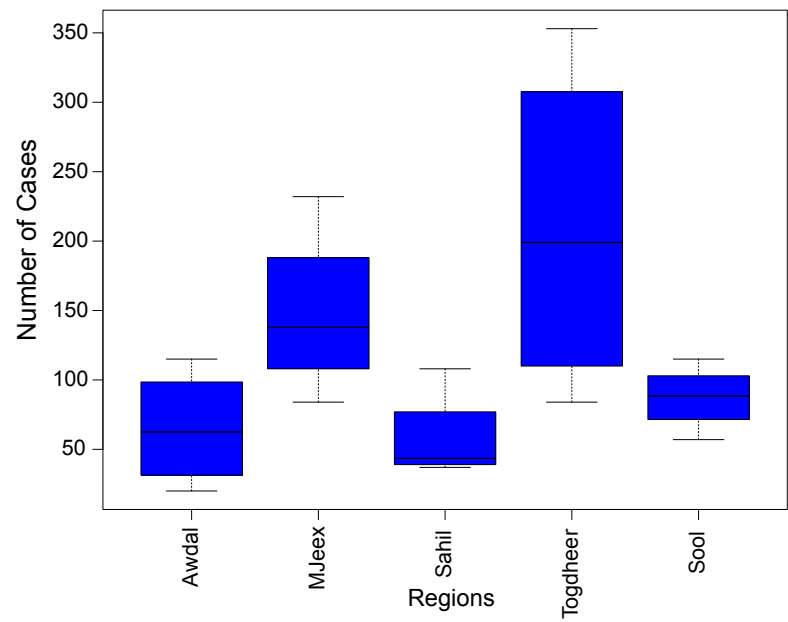

Figure 2: Average number of cases.

\section{Averages by Region from 2011-2014}

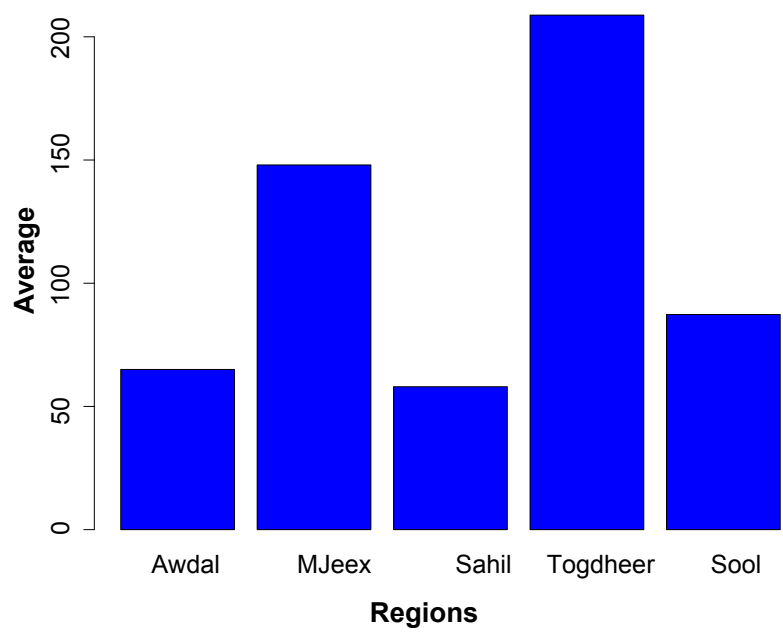

Figure 3: Average number of cases.

children in Somaliland will be investigated. Figures 1-5 show that, there are trend and seasonal effects of the TB infection patterns. We have compared different time series models to select the best model that describes these patterns of the disease and best model is chosen. The best model among the competing models that has captured the dynamics of the disease is Holt-Winters exponential smoothing method.

\section{Additive Holt-Winters exponential smoothing method}

This forecasting method weights the observed time series values unequally taking into account the level, trend and seasonal effects of the data. Exponential smoothing methods have been found to be most effective when the parameters describing the time series may be changing slowly over time. Most recent observations are weighted more heavily than the old observations. Given a time series $y_{1}, y_{2}, \ldots, y_{n}$ that exhibits a linear trend and a seasonal variation that are slowly changing, the estimates of the level $L_{t}$, trend $T R_{t}$ and the seasonal effect $S t$ are as follows

$$
\begin{aligned}
& L_{t}=\alpha\left(Y_{t}-S_{t-L}\right)+(1-\alpha)\left(L_{t-1}+T R_{t-1}\right) \\
& T R_{t}=\beta\left(L_{t}-L_{t-1}\right)+(1-\beta) T R_{t-1} \\
& S_{t}=\delta\left(Y_{t}-L_{t}\right)+(1-\delta) S_{t-1}
\end{aligned}
$$

Where $\alpha \beta$ and $\delta$ are smoothing constants between 0 and 1 and a point forecast made at time $T$ for $\mathrm{Y}_{\mathrm{t}+\tau}$ is $\mathrm{Y}_{\mathrm{t}+\tau}=\mathrm{L}_{\mathrm{t}+\tau} \mathrm{TR}_{\mathrm{t}}+\mathrm{S}_{\mathrm{t}+\tau \mathrm{L}}$, for $\tau=1,2, \ldots$, and the estimates of the above parameters $\hat{\alpha}, \hat{\beta}, \hat{\delta}$, are based on the times series accuracy measures, particularly the mean square error (MSE) and the mean absolute percentage error (MAPE). The MSE and the MAPE are given by $M S E=\frac{1}{n} \sum_{t=1}^{n}\left(y_{t}-\hat{y}_{t}\right)^{2}$ and MAPE $=\left[\frac{1}{n} \sum_{t=1}^{n}\left|\frac{y_{t}-\hat{y}_{t}}{y_{t}}\right|\right] * 100 \%$ respectively. The model that has the minimum forecast errors is chosen to forecast the future trends of the given time series.

\section{Data collection and descriptive analysis}

The data used in this study were collected from five regions in Somaliland from the period of 2011-2014. Tuberculosis surveillance 
data are compiled and recorded in the database center of the planning section at the public health department in the ministry of health. General hospitals in five regions have to report all the new cases of tuberculosis every month to the planning section in the ministry of health. Somaliland has a relatively reliable surveillance and reporting system for infectious diseases compared to other parts of Somalia. We reviewed all the available data about the children under five years old suspected of TB infection stored in Somaliland's monthly ministry of health records in this period. The number of reported suspected cases of TB during this period were 2268 cases.

Figure 1a shows how the yearly averages for the number of suspected TB cases for the children (under five years) is increasing for the last two years, incidence has increased 32\% each year and the number of the reported cases has reached the peak in 2014. Figure $1 \mathrm{~b}$ displays monthly averages for the number of the infected children. Highest cases were reported in March, July and August. Figures 2 and 3 present Boxplots and barcharts of the number of cases by region for the five regions. Table 1 shows the estimated case proportions and their $95 \%$ confidence intervals for the under-five years children suspected of TB infection in the five regions. Four of the regions, namely Maroodi Jeex, Togdheer, Awdal and Sool are inland regions, whereas Sahil is a coastal region. In Sahil region most of khat sessions are held outdoors where the levels of pollutants are less concentrated. During that period, Togdheer region is accounted for the highest proportion of cases $38 \%$

\begin{tabular}{|c|c|c|}
\hline Region & Proportion & $\mathbf{9 5 \%} \mathbf{C l}$ \\
\hline Awdal & 0.115 & $(0.102-0.129)$ \\
\hline Mjeex & 0.261 & $(0.243-0.280)$ \\
\hline Sahil & 0.102 & $(0.090-0.116)$ \\
\hline Togdheer & 0.368 & $(0.348-0.388)$ \\
\hline Sool & 0.154 & $(0.139-0.169)$ \\
\hline
\end{tabular}

Table 1: Under-Five TB Proportions.

\begin{tabular}{|c|c|c|}
\hline Region & Est. Pop. & Est. Per $\mathbf{1 0 0 0 0 0}$ \\
\hline Awdal & 100990 & 258 \\
\hline Mjeex & 1863001 & 442 \\
\hline Togdheer & 108205 & 772 \\
\hline Sool & 49114 & 711 \\
\hline
\end{tabular}

Table 2: Under-Five TB Incidence.

(a)

Tuberculosis

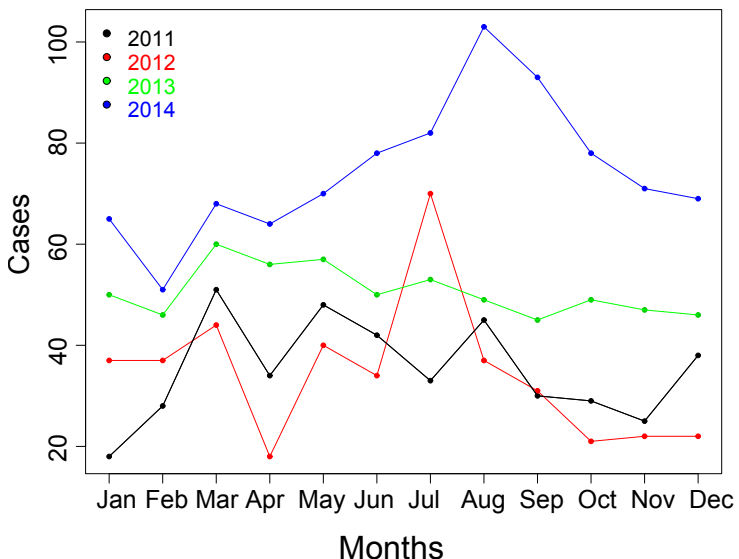

whereas the coastal region of Sahil has lowest $10 \%$, the proportions of the other three regions; Awdal, Maroodi jeex, and Sool are 11.5\%, $26.1 \%$, and $15.4 \%$ respectively.

Table 2 displays the estimated number of under-five year old children in each region and the estimated number of infected cases per 100,000 populations of these children. The incidences during those years for the different regions are 258, 442, 772, and 711 for Awdal, Maroodi jeex, Togdheer, and Sool respectivly. The Population of Sahil region is unknown but included in Maroodi jeex. Incidence rates of Sool region have been in sustained decline over the past four years whereas those rates were increasing for the other four regions.

\section{Predictions}

Figure $4 \mathrm{a}$ depicts the time series plot of the monthly reported cases during those years and Figure $4 \mathrm{~b}$ displays time series plot for the complete data which contain 48 observations. Statistical techniques are utilized to understand the trends of the disease. The best time series model that fits these data is investigated. Many tentative different time series models were fitted and compared by using various accuracy measures and model selection criteria like Mean Absolute Percentage Error (MAPE), Mean Absolute Deviation (MAD), and Mean Square Error (MSE). Based on these criteria, the best model is chosen. After searching the best prediction method for this data series, we found the most appropriate method for the prediction of the changing patterns for this disease in under-five year children as the Additive Holt-Winters Exponential Method with level, trend and seasonal smoothing parameters, $\hat{\alpha}=0.2, \hat{\beta}=0.1, \hat{\delta}=0.01$ respectively. Out of the 48 observations, 45 of them are used to estimate the changing level, trend and seasonal indices and the observations of the last three months were predicted. The predicted values and their prediction intervals are presented in Table 3. Figure 5 depicts the time series plot of the original data with their predicted values.

According to the accuracy measures the model has fitted the data well. Figure 5 shows the plots of the original data and the one-step ahead predictions. These plots clearly show the strength of the employed model. Table 3 is presented the observed, the predicted and the $95 \%$ prediction intervals for the number of cases in the last three months of 2014. The predicted values are very close to the actual observations and (b)

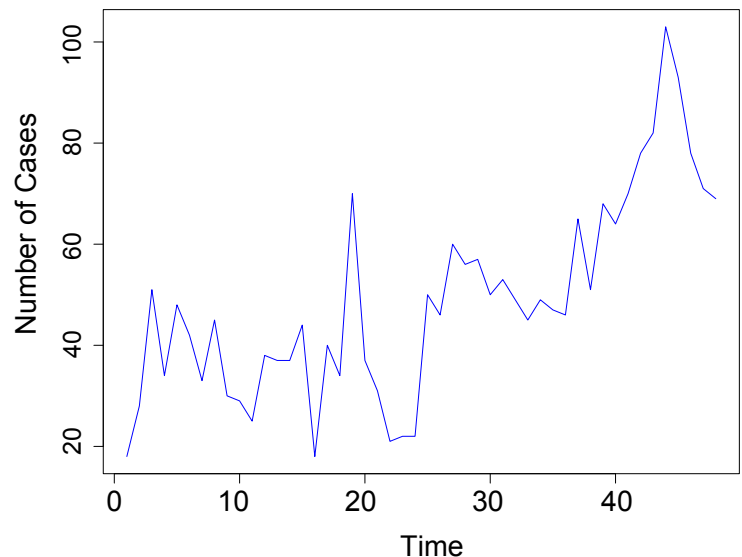

Figure 4: TB Time Series Plots. 4a: Monthly reported cases during those years, 4b: Complete data which contain 48 observations. 


\begin{tabular}{|c|c|c|c|}
\hline Month & Actual & Predicted & $\mathbf{9 5 \%}$ PI \\
\hline Oct. 14 & 78 & 70 & $51.31-89.24$ \\
\hline Nov. 14 & 71 & 70 & $50.69-89.20$ \\
\hline Dec. 14 & 69 & 75 & $55.69-94.87$ \\
\hline
\end{tabular}

Table 3: Three Month TB Prediction

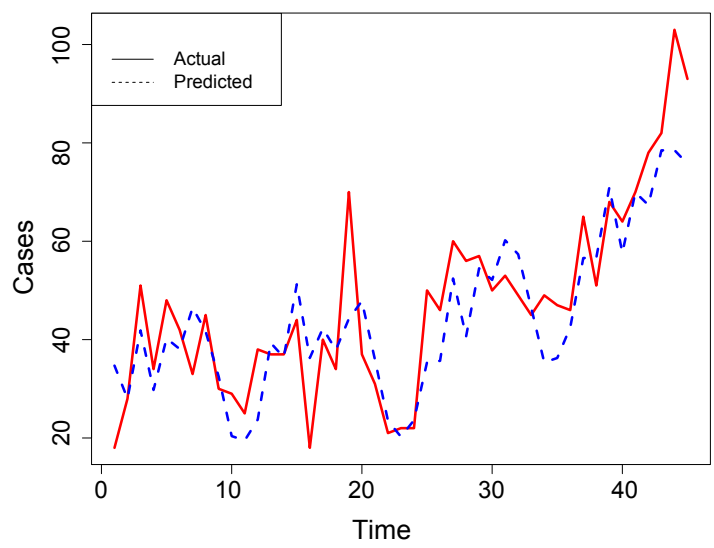

Figure 5: Actual and one-period ahead predictions.

each prediction interval has reasonable length. Based on these results, the best model to be adopted for the short term prediction for the number of the suspected TB cases among the under-five year's children in Somaliland appears to be the Additive Holt-Winters Exponential Smoothing Method.

\section{Conclusions and Paradigm Shift for Tuberculosis Control in Children}

Current global tuberculosis (TB) control paradigm mainly focuses on cutting transmission through early case detection and effective treatment [18]. Social and environmental interventions are not usually delivered or funded by the health sectors [1]. Poor sanitation and overcrowding take the environment more propitious for the breeding of mycobacterium tuberculosis. The most productive breeding grounds of the Mycobacterium tuberculosis are neglected and did not get sufficient attention and enough funding. A key similarity between vector-borne and air-borne diseases is the existence of the breeding grounds for both diseases. For example, the spread of vector-borne diseases is highly correlated with precipitation where as one of the major risk factors for airborne diseases is overcrowding and unventilated buildings. Given the promising results in fighting vector-borne diseases, it is necessary to adopt similar approaches in fighting against the spread of TB. Those approaches included the Roll Back Partnership initiative. The partnership comprises hundreds of partners organized in different constituencies like governmental, nongovernmental, communitybased organizations, foundations, and academic institutions. Those initiatives helped to achieve substantial successes against fighting vector-borne diseases and created conditions that have positive impact on the dynamics of these diseases. The risk of exposure is determined by the physical environment in which the contact takes place, including aspects of crowding, air flow and humidity [35]. Environmental management strategies and community action helped the control measures in targeting towards the most productive breeding sites. For example, stagnant water where mosquito larvae grow was treated with chemicals that have substantially reduced its population. Same approaches could be adopted in combating the spread of tuberculosis. These approaches could include, identifying the most productive
Mycobacterium tuberculosis breeding sites, and then using local community and government clean-up campaigns in eradicating these breeding sites. Clearly, health care professionals in partnership with the local community and the government can easily undertake the empowerment activities to build public awareness for the risks of the disease. Promoting the spirit of joint cooperation between health care, local community and the government could help to implement the intervention and mitigation measures to combat the spread of the disease. Those groups together could provide comprehensive monitoring at the breeding grounds. A prevention and control plan designed to contain Mycobacterium tuberculosis breeding and to treat the infected promptly could produce good results and substantially reduce the spread of the disease. Such a plan needs to give an opportunity to the communities and the local authorities to provide their input in fighting with the disease, and to bring different groups together to fight the disease on multiple fronts. Local communities could be used to identify the most productive Mycobacterium tuberculosis breeding sites, whereas community organizations can play the active role of raising the awareness of the patients and groups within the community who may be at higher risk of TB infection. Those groups could benefit from health promotion and disease prevention programs targeted to their specific needs. On the other hand, governments can actively engage in building health safety standards, improve sanitation and hygiene management. Governments can also support media campaigns to increase awareness, community empowerment, education, and able to enforce environmental laws and promote compliance [36-40].

Prompt reporting of adult cases and contact investigations would be very difficult unless all breeding grounds are located, supervised and controlled. Since TB is a social disease that requires a life-course approach, existing interventions could be broadened by pursuing a lifecourse framework that gives more consideration on structural, social, cultural and environmental contexts. Identification of the crowding sites need the consideration of a wide range of social, structural environmental factors and the social conditions that shape the burden of infectious diseases in certain populations. These determinants would contribute in understanding the social interactions that promote the transmission of the disease. Locating breeding areas and high risk groups in these places would help to target strategies for early detection of people in need of treatment.

In Somalia, medical professionals believe the majority of the TB breeding grounds in the country are the poorly ventilated overcrowded Marfishes that people gather to chew khat. Since the customers of these marfishes stay long hours in overcrowded khat chewing houses, they are at a higher risk of getting infected and most of them share their young children in a master bedroom. Thus, TB infection control plan designed to ensure prompt detection of infectious patients and protecting the very young, must include health monitoring and surveillance systems directed to these places. Concentrating more focus on these sites would help to improve national health monitoring and surveillance systems. Without the consideration of these breeding grounds, the long-term epidemiological goals for global TB control in general and in Somalia in particular will be difficult to be achieved. Therefore, to contain the spread of the disease to the too young and the most vulnerable children, local communities and the different levels of the government must be completely involved in the intervention and the preventive activities and more resources have to be allocated. The failure to trace and appropriately manage adult source cases in these breeding sites will make difficult to prevent and control TB from Somali children.

\section{References}

1. WHO (2016) WHO calls on countries and partners to "Unite to End Tuberculosis". Media centre, WHO. 
Citation: Hassan M (2018) Trends of Tuberculosis in Somaliland's Young Children after the Conflict and the Role Khat Marfishes Play Its Transmission. J Biom Biostat 9: 397. doi: 10.4172/2155-6180.1000397

2. Centers for Disease Control and Prevention (1994) Estimates for future global tuberculosis and mortality. MMWR Morb Mortal Wkly Rep 42: 961-964.

3. Diel R, Loddenkemper R, Nienhaus A (2016) Consequences of tuberculosis among asylum seekers for health care workers in Germany. J Occup Med Toxicol 11: 4.

4. Lillebaek T, Dirksen A, Baess I, Strunge B, Thomsen VO (2002) Molecular evidence of endogenous reactivation of Mycobacterium tuberculosis after 33 years of latent infection. J Infect Dis 185: 401-404.

5. Svensson E, Millet J, Lindqvist A, Olsson M, Ridell M (2011) Impact of immigration on tuberculosis epidemiology in a low-incidence country. Clin Microbiol Infect 17: 881-887.

6. Gustafson P, Lisse I, Gomes V (2007) Risk factors for positive tuberculin skin test in Guinea Bissau. Epidemiology 18: 340-347.

7. Raviglione MC, Snider DE, Kochi A (1995) Global epidemiology of tuberculosis. Morbidity and mortality of a worldwide epidemic. JAMA 273: 220-226.

8. World Health Organization (2006) Guidance for National Tuberculosis Programmes on the Management of Tuberculosis in Children WHO/HTM/ TB/2006.371.

9. Gomes VF, Andersen A, Wejse C (2011) Impact of tuberculosis exposure at home on mortality in children under 5 years of age in Guinea-Bissau. Thorax 66: $163-167$

10. Hesseling AC, Schaaf HS, Gie RP (2002) A critical review of diagnostic approaches used in the diagnosis of childhood tuberculosis. Int J Tuberc Lung Dis 6: 1038-1045.

11. CDC-TB-Tuberculosis in Children in the United States 2014.

12. Lobato MN, Mohle-Boetani JC, Royce SE (2000) Missed opportunities for preventing tuberculosis among children younger than five years of age. Pediatrics 106/6/ E75.

13. Donald PR (2004) Childhood tuberculosis: the hidden epidemic. Int J Tuberc Lung Dis 8: 627-629.

14. Lienhardt C, Sillah J, Fielding K (2003) Risk factors for tuberculosis infection in children in contact with infectious tuberculosis cases in the Gambia, West Africa. Pediatrics 111: 608-614.

15. Jenkins HE, Tolman AW, Yuen CM (2014) Incidence of multidrug-resistan tuberculosis disease in children: systematic review and global estimates. Lancet 383: 1572.

16. Murray CJL, Styblo K, Rouillon A (1990) Tuberculosis in developing countries: burden, intervention and cost. Bull Int Union Tuberc Lung Dis 65: 624

17. Lewinsohn DA, Gennaro ML, Scholvinck L (2004) Tuberculosis immunology in children: diagnostic and therapeutic challenges and opportunities. Int J Tuberc Lung Dis 8: 658-674.

18. Sindani I, Fitzpatrick C, Falzon D (2013) Multidrug-resistant tuberculosis somalia 2010-2011. Emerging Infectious Diseases 19: 478-480.

19. Wilkinson R, Marmot M (2003) Social Determinants of Health: The Solid Facts 2nd edn. Denmark: World Health Organization,

20. Van Zwandenberg DF (1960) The influence of the number of bacilli on the development of tuberculosis disease in children. Am Rev Respir Dis 82: 31-44.
21. Singh J, Sankar MM, Kumar S (2013) Incidence and prevalence of tuberculosis among household contacts of pulmonary tuberculosis patients in a peri-urban population of South Delhi, India. PLoS ONE 8: e69730.

22. Goudie AJ (2002) Importing khat, legal but dangerous (letter). Lancet 2: 13401341.

23. Drucker E, Alcabes $P$, Bosworth W, Sckell B (1994) Childhood tuberculosis in the Bronx, New York. Lancet 343: 1482-1485.

24. Hawker J, Bakhshi S, Ali S, Farrington P (1999) Ecological analysis of ethnic differences in relation between tuberculosis and poverty. BMJ 319: 1031-1034.

25. Mangtani P, Jolley D, Watson JM, Rodrigues LC (1995) Socioeconomic deprivation and notification rates for tuberculosis in London during 1982-91. BMJ 310: 963-966.

26. Bhatti N, Law MR, Morris JK, Halliday R, Moor-Gillon J (1995) Increasing incidence of tuberculosis in England and Wales: a study of likely causes. BM 310: 967-969.

27. Baker M, Das D, Venugopal K, Howden-Chapman P (2008) Tuberculosis associated with household crowding in a developed country. J Epidemiol Community Health 62: 715-721.

28. Schmidt CW (2008) Linking TB and the Environment: An overlooked mitigation strategy. Environ Health Perspect 116: A478-A485.

29. Chapman JS, Dyerly MD (1964) Social and other factors in intra-familia transmission of tuberculosis. Am Rev Respir Dis 90: 48-60.

30. Cao S, Wang F, Tam W, Tse LA, Kim JH, et al. (2013) A hybrid seasonal prediction model for tuberculosis incidence in China. BMC Med Inform Decis Mak 13: 56.

31. Kam HJ, Sung JO, Park RW (2010) Prediction of daily patient numbers for a regional emergency medical center using time series analysis. Healthc Inform Res 16: 158-165.

32. Li N, Yin F, Lix X (2010) Study on the time series analysis in forecasting the incidence of tuberculosis in Sichuan province. Modern Prevent Med 37: 14261428

33. Yi J, Du CT, Wang RH, Liu L (2007) Applications of multiple seasona autoregressive integrated moving average (ARIMA) model on predictive incidence of tuberculosis. Chin J Prev Med 41: 118-121.

34. Zhang X, Zhang T, Young AA (2014) Applications and comparisons of fou time series models in epidemiological surveillance data. PLoS One 9: e88075.

35. Rieder H (1999) Epidemiologic basis of tuberculosis control. Paris: Internationa Union Against Tuberculosis and Lung Disease (1stedn), pp: 1-162.

36. Centers for Disease Control (1989) A strategic plan for the elimination of tuberculosis. MMWR Suppl 38: 1s-25s.

37. Lnnroth K, Jaramillo E, Williams BG, Dye C, Raviglione M (2009) Drivers of tuberculosis epidemics: the role of risk factors and social determinants. Soc Sci Med 68: 2240-2246.

38. WHO (2018) Tuberculosis. Media centre, WHO

39. WHO (2011) Social determinants of health. Gender, equity and human rights, $\mathrm{WHO}$.

40. WHO (2015) Global Tuberculosis Report (20thedn), WHO. 